\title{
AN IDENTITY IN ARITHMETIC
}

\author{
BY H. D. BLOCK AND JACOB MARSCHAK
}

Communicated by Mark Kac, January 20, 1959

During the course of a study ${ }^{1}$ on Theories of Choice ${ }^{2}$ the authors encountered the following arithmetical identity which appears to have some independent mathematical interest. Let $N$ denote the set of integers $\{1,2, \cdots, n\}$; let $M$ be a fixed subset of $N$ and $i$ a fixed element of $M$. For any permutation $r$ of the set $N$ let $i_{r}$ denote that integer of $N$ which is ranked in the $i$ th position by the permutation $r$. Let $R(i, M)$ denote the set of those permutations which rank $i$ before the other elements of $M$. Let $n$ positive numbers $u_{1}, u_{2}, \cdots$, $u_{n}$ be given. Then we have the identity

$$
\left(\prod_{j=1}^{n} u_{j}\right) \sum_{r \in R(i, M)} \prod_{k=1}^{n}\left[\sum_{i=k}^{n} u_{i_{r}}\right]^{-1}=u_{i} / \sum_{j \in M} u_{j} .
$$

From the nature of the functions appearing on each side (1), or from the proof, it is clear that the numbers $u_{k}$ need not be restricted to be positive; indeed they could be any real or complex numbers, or elements of an arbitrary field, provided that no subset has zero sum.

The identity (1) becomes intuitively clear from the following probability considerations. Consider an urn with $n$ coins, numbered $1,2, \cdots, n$; the size of the $k$ th coin being $u_{k}$. The probability of a coin being chosen is proportional to its size. Suppose all $n$ coins are successively drawn without replacement. The probability that the order in which they appear is a particular permutation $r$, is $\prod_{k=1}^{n} u_{k_{r}} / \sum_{j=\mathbb{t}}^{n} u_{j_{r}}$, using conditional probabilities. Summing over all permutations of $R(i, M)$ we see that the left side of (1) represents the marginal probability that $i$ precedes the rest of $M$, disregarding the elements of $N-M$. On the other hand if we initially remove from the urn those coins whose numbers are the elements of $N-M$, then the probability that $i$ is chosen first from the set $M$ is given by the right side of (1).

The authors felt at first that these probability considerations furnished a proof of (1) in virtue of some theorem in measure theory which assured that the two probabilities mentioned above must be

${ }^{1}$ Research undertaken by the Cowles Commission for Research in Economics under a contract with the Office of Naval Research.

${ }^{2}$ Cowles Foundation Discussion Paper No. 66: Random orderings and stochastic theories of responses, by H. D. Block and J. Marschak, mimeographed. To be published in honor of $\mathrm{H}$. Hotelling. 
equal. We were however, unable to find such a theorem and resorted to an induction proof as follows. First we show, by induction on $n$ that

$$
\sum^{(1)} \prod_{k=1}^{n} \frac{u_{k_{r}}}{\sum_{j=k}^{n} u_{j_{r}}}=1
$$

where $\sum^{(1)}$ is taken over all permutations $r$ of the set $N$. This follows from the fact that the left side of (2) is equal to $\left(\sum_{\alpha=1}^{n} u_{\alpha} / \sum_{\beta=1}^{n} u_{\beta}\right)$ $\sum^{(2)} \prod_{k=2}^{n} u_{k_{r}} / \sum_{j=k}^{n} u_{j_{r}}$, where $\sum^{(2)}$ is taken over all permutations of the set $\{N-(\alpha)\}$. (This is obtained by grouping all those permutations $r$ such that $1_{r}=\alpha$, and then summing on $\alpha$ ). Next we show, by induction on $n$, that for any subset $M$ of $N$ and any permutation $s=\left(1_{s}, \cdots, m_{s}\right)$ of $M$ :

$$
\sum^{(3)} \prod_{k=1}^{n} \frac{u_{k_{r}}}{\sum_{j=k}^{n} u_{j_{r}}}=\prod_{k=1}^{m} \frac{u_{k_{s}}}{\sum_{j=k}^{m} u_{j_{\varepsilon}}}
$$

where $\sum^{(3)}$ is taken over all permutations $r$ of $N$ which preserve the order $1_{8}, 2_{8}, \cdots, m_{8}$ of the subset $M$. We omit the details of this lemma here. The identity (1) is obtained from (3) by setting $i=1_{s}$ and summing over all permutations of the set $\{M-(i)\}$, using equation (2).

Cornell Universtty,

YALE UNIVERSITY AND

Carnegie Institute of Technology 